\title{
In situ and real-time probing of quasicrystal solidification dynamics by synchrotron imaging
}

\author{
H. Nguyen Thi, ${ }_{1}$ J. Gastaldi, ${ }^{2}$ T. Schenk,${ }^{3}$ G. Reinhart, ${ }^{1}$ N. Mangelinck-Noel,${ }^{1}$ V. Cristiglio, ${ }^{3}$ B. Billia, ${ }^{1}$ B. Grushko, \\ J. Härtwig, ${ }^{3}$ H. Klein, ${ }^{5}$ and J. Baruchel ${ }^{3}$ \\ ${ }^{1}$ L2MP, UMR CNRS 6137, Université Paul Cézanne-Aix-Marseille III, Faculté des Sciences de Saint-Jérôme, Case 142, \\ 13397 Marseille Cedex 20, France \\ ${ }^{2}$ CRMCN-CNRS (UPR 7251), Campus Luminy, Case 913, 13288 Marseille Cedex 09, France \\ ${ }^{3}$ ESRF, BP 220, 38043 Grenoble, France \\ ${ }^{4}$ IFF, Forschungszentrum Jülich GmbH, 52425 Jülich, Germany \\ ${ }^{5}$ Laboratoire de Cristallographie, CNRS Bât. F, BP 166, 38402 Grenoble Cedex 9, France \\ (Received 19 July 2005; revised manuscript received 17 July 2006; published 15 September 2006; \\ publisher error corrected 18 September 2006)
}

\begin{abstract}
Quasicrystal growth remains an unsolved problem in condensed matter. The dynamics of the process is studied by means of synchrotron live imaging all along the solidification of icosahedral AlPdMn quasicrystals. The lateral motion of ledges driving faceted growth at the solid-melt interface is conclusively shown. When the solidification rate is increased, nucleation and free growth of new faceted grains occur in the melt due to the significant interface recoil induced by slow attachment kinetics. The detailed analysis of the evolution of these grains reveals the crucial role of aluminum rejection, both in the poisoning of their growth and driving fluid flow.
\end{abstract}

DOI: 10.1103/PhysRevE.74.031605

\section{INTRODUCTION}

Quasicrystals are complex metallic alloys that display long-range orientational order with symmetries (fivefold, eightfold,...) incompatible with periodicity, previously considered as strictly forbidden. They also exhibit many specific properties (high hardness, low electric conductivity, low friction coefficient,...) that have motivated engineering efforts to use them as new materials (e.g., for advanced surface coatings or catalysis). The main challenge of quasicrystal physics is still to elucidate how the quasiperiodic order can extend up to the centimeter size of the grains routinely grown nowadays [1-3]. Whether the formation of the stable quasicrystal structure is constrained by local growth rules [4] or by the establishment of long-range atomic correlation [5] is still undetermined. Because translation symmetry is lacking, it seems unlikely that quasicrystals build up by the attachment of single atoms, thereby generating the whole network just like crystals do. As icosahedral clusters have been recently identified in quasicrystal-forming liquids [6], the idea of growth by the attachment of these clusters at the liquid-solid interface [7] is reviving. Experimental evidence that quasicrystals grow by the forward movement of facets can be found in the literature, such as the post-mortem observation of grains displaying facets $[8,9]$. Such features were not accessible in the direct observation of rapid solidification at high liquid undercooling [10], as growth was dendritic. Striking but still indirect aftergrowth indication is also provided by the shape of internal voids analyzed as negative quasicrystals [11]. Yet, this situation actually corresponds to growth from the vapor, and not to solidification from the melt. Furthermore, asymmetry is long recognized between solidification and melting, in particular for systems with slow growth kinetics $[12,13]$.

It is therefore critical and timely to deepen the understanding of the dynamics of quasicrystal formation and growth from the liquid alloy. Indeed, it is more than likely
PACS number(s): 81.10.Aj, 61.44.Br, 68.55.-a, 81.30.Fb

that clarifying the growth mechanisms, in which the peculiar quasicrystal structure is continuously constructed, will in a timely basis bring out critical elements to elucidate how nature in practice succeeds to propagate "forbidden" symmetries. In order to get a clear insight on the growing grains and the detailed morphology of the solid-liquid interface we carried out the first live probing of the solidification of icosahedral AlPdMn quasicrystals. This characterization of the dynamical evolution of the quasicrystal grains is reported in the present paper.

\section{EXPERIMENT}

Observations were performed by in situ and real time synchrotron x-ray radiography during upward Bridgman solidification, at the ID 19 beamline of the European Synchrotron Radiation Facility (ESRF, Grenoble, France). Radiographs were obtained directly from the monochromatic incident x-ray beam after it has passed through a thin sample solidifying between two graphite foils. Owing to the highly coherent beam delivered by the ESRF synchrotron source [14], these images displayed both absorption and phase contrasts. They were recorded either continuously with a CCD camera [15] or at intervals on high resolution films. Thereby, we were able to image with a large field of view $\left(15 \times 6 \mathrm{~mm}^{2}\right)$ the dynamical phenomena that took place all along the solidification of icosahedral AlPdMn from the melt (Fig. 1). This approach is obviously limited by the resolution of synchrotron radiography, a few micrometers. In particular, complementary transmission electron microscopy would provide direct information on the physical mechanisms at the nanometer scale but with a very narrow field of view, as achieved for instance for semiconductors and oxides [16]. In practice, these mechanisms can opportunely be investigated through their macroscopic manifestations, on the basis of the firmly established and long recognized knowledge available in growth from the melt, or solution, and in solidification processing of alloys. 

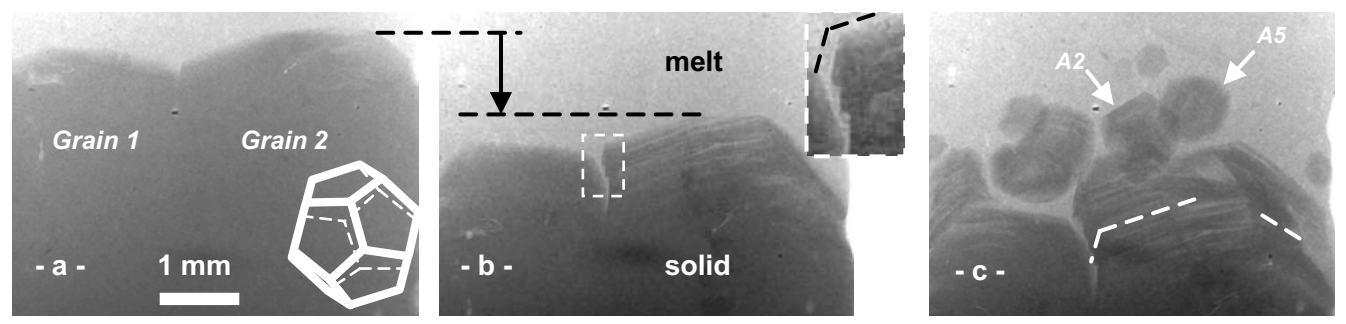

FIG. 1. In situ and real-time x-ray radiographs of AlPdMn quasicrystals growing from the melt: (a) at the end of a growth sequence at a pulling rate $V=0.4 \mu \mathrm{m} / \mathrm{s}$; (b) and (c) during the growth sequence at $V=3.6 \mu \mathrm{m} / \mathrm{s}$. The arrow in (b) shows the front recoil due to the increase of the pulling velocity. The dashed lines in the insert of (b) and in (c) are to guide the eyes in seeing the striations, and allow orientation on Grain 2 by referring to the dodecahedron on (a).

The Bridgman directional solidification setup allowed independent control of both the pulling velocity and the temperature gradient [17]. Two $700 \mu \mathrm{m}$-thick sheets, $40 \mathrm{mms}$ long and $6 \mathrm{mms}$ wide, were prepared by grinding rods of $\mathrm{Al}_{72.4} \mathrm{Pd}_{20.5} \mathrm{Mn}_{7.1}$ alloy, whose composition is known to give the icosahedral quasicrystal phase [1]. These sheets were first melted, and then solidified at various pulling velocities $(0.4-3.6 \mu \mathrm{m} / \mathrm{s})$ under a temperature gradient of $35 \mathrm{~K} / \mathrm{cm}$. Quasicrystallinity of the solid structure was repeatedly checked by indexing Laue patterns recorded after cooling. Energy-dispersive spectra were used for supplementary control, to check if the measured composition was corresponding to icosahedral AlPdMn on the phase diagram.

Indeed, it follows from the Al-Mn-Pd phase diagram that the solidification of icosahedral AlPdMn is noncongruent, with rejection of aluminum $[18,19]$. Striking and unambiguous experimental proof of noncongruent solidification is provided by synchrotron x-ray radiography, namely the good contrast between solid and melt in the radiographs (Fig. 1). Indeed, this good contrast with solid darker than liquid means that the solid is more absorbing the x-ray beam because it is denser, due to lower concentration in light element. Conversely, radiographs taken during congruent solidification from the melt of $\mathrm{Al}_{3} \mathrm{Mg}_{2}$ show almost no contrast between solid and liquid [Fig. 2(a)], and nothing is visible on primary images for noncongruent dendritic solidification of Al-7 wt. \% Si [Figs. 2(b) and 2(c)]. In the latter case, the
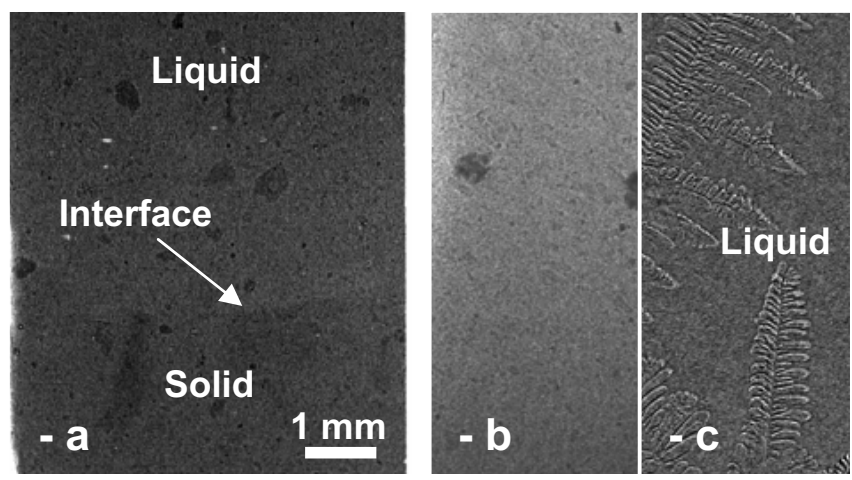

FIG. 2. Synchrotron $x$-ray radiographs of: (a) congruent solidification of $\mathrm{Al}_{3} \mathrm{Mg}_{2}$ from the melt at $V=5 \mu \mathrm{m} / \mathrm{s}$; (b) dendritic growth of Al-7 wt. \% Si by cooling down at $1 \mathrm{~K} / \mathrm{min}$. (c) shows the dendritic microstructure in (b), made visible by image processing. difference in absorption between $\mathrm{Al}$ and $\mathrm{Si}$ is too weak although Si rejection upon solidification is large (Si concentration in solid/Si concentration in liquid $=0.13$ at the solidliquid interface).

Energy dispersive spectra furthermore confirm that aluminum is rejected upon solidification of icosahedral AlPdMn. The composition of the starting alloy, which was produced many times with good reproducibility, is well established at $\mathrm{Al}_{72.4} \mathrm{Pd}_{20.5} \mathrm{Mn}_{7.1}$. Lower $\mathrm{Al}$ concentration is repeatedly found in our after-growth energy dispersive spectroscopy (EDS) measurements of the composition of AlPdMn quasicrystal grains. For instance, the composition of Grain 1 is measured at $\mathrm{Al}_{68.5} \mathrm{Pd}_{23} \mathrm{Mn}_{8.5}$, i.e., $67.8 \leqslant \mathrm{Al} \leqslant 69.2$ at. $\%$. On the average, the $\mathrm{Al}$ concentration deduced from measurements on several grains in three samples is 68.5 at. \% with a standard deviation of 0.5 at. \%, which is significantly less than the minimum of 71.7 at. \% Al in the starting material, obtained by applying the upper relative EDS error bar of $1 \%$.

\section{RESULTS}

In situ x-ray imaging [20] unambiguously unveils the growth dynamics of the faceted solid-melt interface in quasicrystal solidification (Fig. 1, see also Figs 4 and 5). In steady-state growth at $0.4 \mu \mathrm{m} / \mathrm{s}$ [Fig. 1(a)], two grains are growing simultaneously upwards, and the interface between the liquid and these grains shows a cusp at the level of the grain boundary (radiographs basically show projections along the incident $\mathrm{x}$-ray beam). From the outline of the solidmelt interface, facets and facet edges can be identified on grain 1 and grain 2 , which can be described as irregular dodecahedra extending in the direction of pulling [the dodecahedron superposed on Fig. 1(a) gives the orientation of grain 2]. Because the grain shapes are representative of the relative growth rates of the feasible facets, it happens that the dodecahedra are partly truncated.

Then, as the applied pulling velocity is increased to $3.6 \mu \mathrm{m} / \mathrm{s}$, a solidification transient takes place in which the growth velocity and grain shape progressively adapt. After about $600 \mathrm{~s}$, the solidification front has globally receded to a lower temperature [Fig. 1(b)], and the growth rate reached the new pulling velocity (Fig. 3). Concomitantly, the cusp has evolved into a wide and deep liquid groove and, while advancing, the facets have developed both striations running 


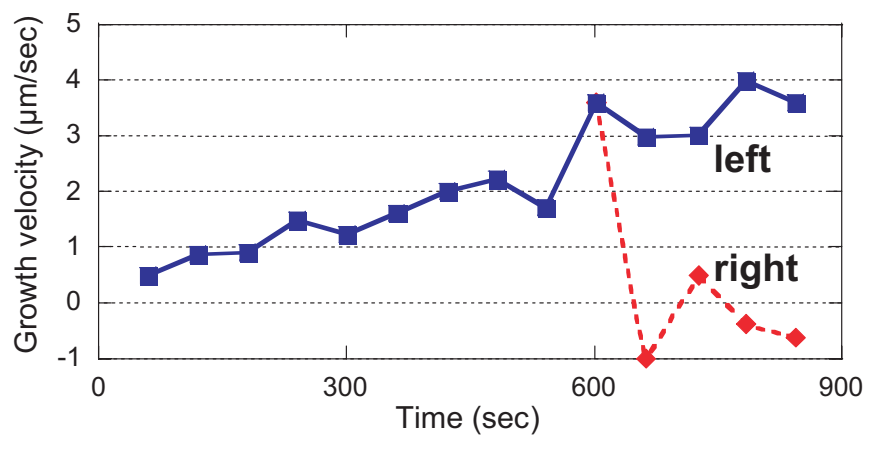

FIG. 3. (Color online) Evolution of the growth velocity of grain 1 (ם) in the solidification transient following the abrupt increase of the applied pulling rate from to 0.4 to $3.6 \mu \mathrm{m} / \mathrm{s}$. The dashed curve ( ) shows the rapid blockage, visible in Fig. 1(c), of the growth of the right part of grain 1 by a newly nucleated grain.

parallel to the solid-liquid interface and notches neatly visible on the left side of grain 2 [see inset in Figs. 1(b) and 1(c)]. Striations delineate arrests of macroscopic bunches of ledges, which merely appear as notches when viewed from the side. When there is enough space available in the liquid, notches can become exacerbated and even evolve into prominent overhangs as shown in Fig. 4, taken from another solidification run at $V=1.2 \mu \mathrm{m} / \mathrm{s}$ on grain 1 and grain 2 of Fig. 1. This stage with a large liquid groove is not reached in Fig. 1(c), one reason being the formation of new quasicrystals in front of grain 1 and grain 2 at this pulling rate. Indeed, small faceted quasicrystals nucleate and grow in the melt just ahead of grains 1 and 2 [Fig. 1(c)], about $60 \mathrm{sec}$ after Fig. 1(b). Mutual impediment of growth is observed in Fig. 1(c) as grains approach each other. This blockage is seen to effectively begin with the screening of grains 1 and 2 (Fig. 3).

Figures 5(a)-5(c) focus on the competitive growth of new grains. They show that the growth of each grain progressively interferes with that of neighbors. The velocity of each

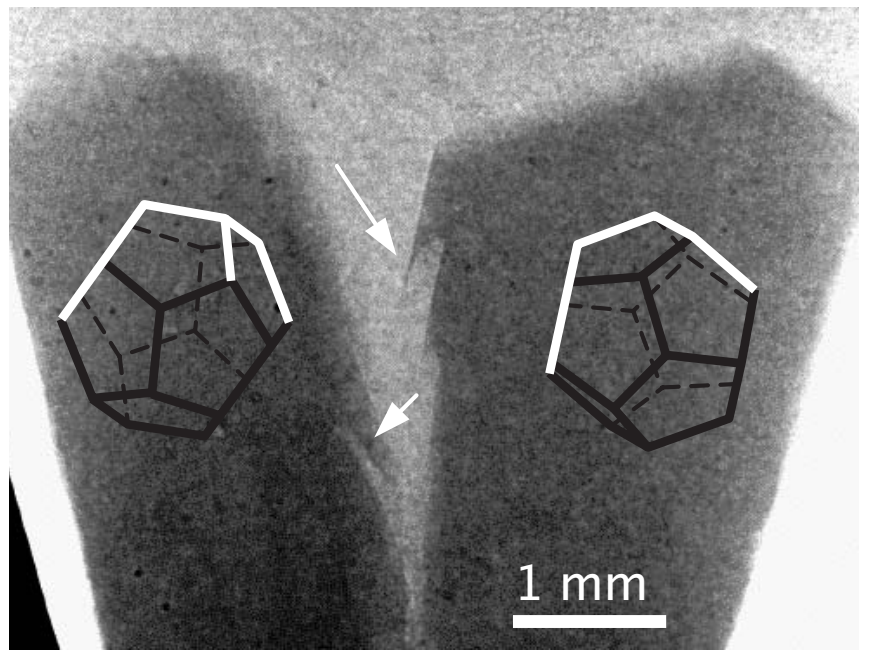

FIG. 4. Radiograph of the two $i$-AlPdMn grains in Fig. 1 achieving coupled growth in the transient following the application of pulling at $1.2 \mu \mathrm{m} / \mathrm{s}$. Prominent overhangs (arrows) form in the wide liquid groove. The grain caps actively growing are marked in white on the dodecahedra.

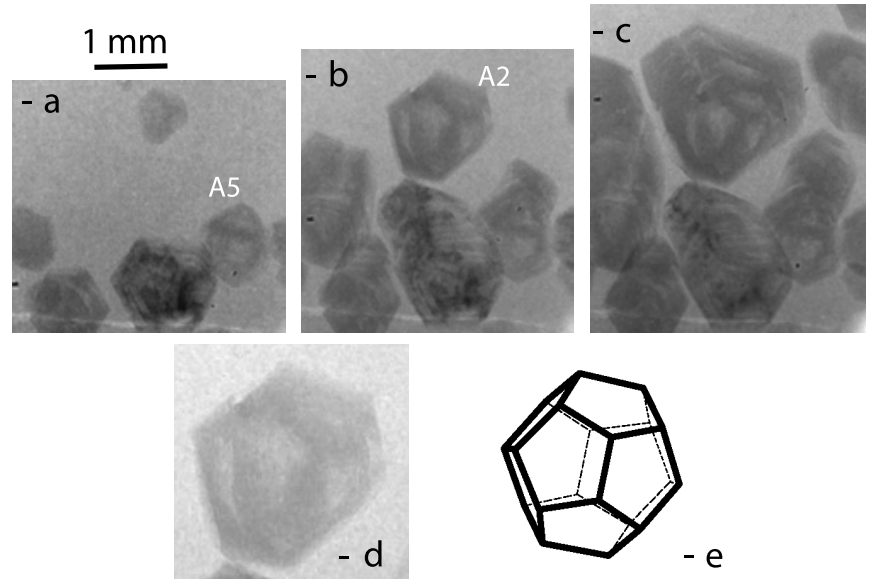

FIG. 5. Typical evolution of a free grain after nucleation in the melt, showing progressive blockage by neighbors: (a) $180 \mathrm{sec}$, (b) $421 \mathrm{sec}$, (c) $843 \mathrm{sec}$, after nucleation. Pulling rate $V=3.6 \mu \mathrm{m} / \mathrm{s}$. The grain orientation deduced from radiograhy contrasts in (d) is shown by the projected dodecahedron (e).

facet or edge making the outline of a free grain can be measured along the normal to its trace on the radiographs (Fig. 6). All the facet/edge velocities initially increase with time, and finally fall down. At short times, the growth of the new grain is free but not isotropic, which is most obvious for the edges propagating upwards and downwards (arrows 1 and 6 , respectively). When a facet $\left(V_{3}\right.$ to $\left.V_{5}\right)$, or an edge $\left(V_{6}\right)$, is approaching a neighbor grain, its growth progressively feels that of the grain due to the increasing overlap of the diffusion fields. Eventually, its velocity reaches zero upon impingement. Meanwhile, facet 2 and edge 1, which see open melt ahead as they grow at a small angle to the pulling direction,

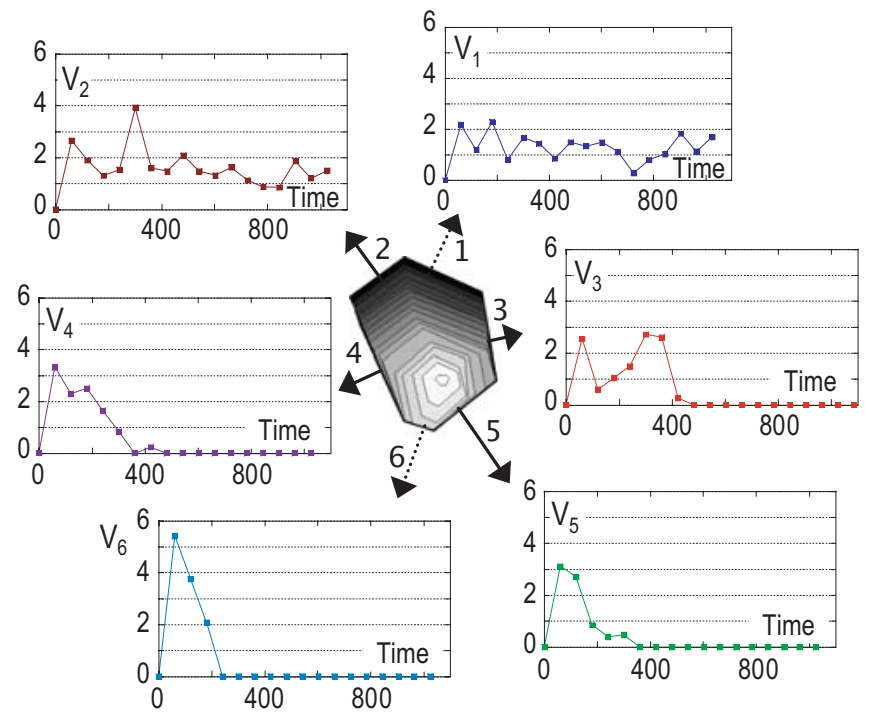

FIG. 6. (Color online) Variation with time ( $\mathrm{sec})$ of the velocity $\left(\mu \mathrm{m} / \mathrm{sec}\right.$ ) of the facets (arrows 2 to 5 , velocities $V_{2}$ to $V_{5}$ ) and edges (arrows 1 and 6, velocities $V_{1}$ and $V_{6}$ ) making the outline of the highest grain in Fig. 5(a) (see also video 2 in Ref. [20]). The origin of time is at grain nucleation, $540 \mathrm{~s}$ after applying pulling at $3.6 \mu \mathrm{m} / \mathrm{s}$. 
conversely continue to advance. This stage, which may even reach a steady-state as indicated by the plateaus in $V_{1}$ and $V_{2}$ (Fig. 6), results in grain elongation [Figs. 5(b) and 5(c)]. The dodecahedron shape is preserved as long as the grain grows freely. Indeed, while grains are impinging, the facets facing each other undergo progressive smoothening that gives way to curved boundaries. As long as icosahedral AlPdMn grains are growing, aluminum is rejected in the surrounding melt. Contrary to latent heat, aluminum cannot be extracted through the existing solid and the crucible walls so that it can only accumulate in the melt in the spaces separating the grains, which are narrowing with time. This gives a solutal character to the blockages observed in Figs 1(c) and 5. Indeed, such blocking phenomena due to the rejection of chemical specie(s) are very common and well documented in casting, where they lead to the columnar-equiaxed transition (CET), and limit the growth of equiaxed grains [21-23]. Qualitatively, the blocking in Fig. 1(c) of the columnar quasicrystal grains 1 and 2 by the new grains growing just ahead is basically similar to the regular CET, and the growth/ competition of the new quasicrystal grains in Fig. 5 is very much alike the growth/competition of the equiaxed grains in casting.

Moreover, the black contrasts decorating new grains are worth noticing [Figs. 1(c) and 5(a)-5(c)]. In practice, the geometry of these contrasts also enable the determination of grain orientation [Figs. 5(d) and 5(e)], as done for grain 2 in Fig. 1 at an early stage of its development. This method is somewhat similar to the one used to orient dendritic icosahedral AlMn grains from optical metallographs [24]. The new faceted grains are dodecahedral polyhedra often oriented with either a twofold (e.g., $A 2$ in Figs. 1(c) and 5(b)] or fivefold axis (e.g., A5 in Figs. 1(c) and 5(a)]) along the incident $\mathrm{x}$-ray beam. A threefold axis is more rarely observed.

\section{DISCUSSION}

The striations and notches evidenced in x-ray radiography (Fig. 1) have to be related to ledge growth (lateral motion of macrosteps, or bunches of macrosteps). Indeed, it is well documented and long recognized, in particular for solution and melt growth, that these features result from the repeated kinetic instability of facets, leading to the development of skeletal shapes $[25,13]$. The scenario of facet instability is the following. Ledges are generated at facet edges and vertices where undercooling and solid-liquid interface roughness are locally higher. In their lateral motion, these ledges scrap the chemical specie(s) rejected in the melt upon solidification down over the facets. This process has a selfpoisoning effect on ledge spreading, eventually until complete inhibition. It is of value to note that models based on normal growth with "thick" diffuse interface region $[4,5,7]$ are not suited to describe ledge growth. Besides, were quasicrystal solidification controlled by local heat or solute flow, the growth habit would resemble to the shapes observed in the isothermal dissolution of alloys. For instance, the difference in growth habit between solidification controlled by local heat or solute flow, i.e., limited by the rate at which latent heat or rejected alloying elements can be removed from the region of the interface, and solidification controlled by ledge spreading can be illustrated by considering the isothermal solidification and dissolution (Fig. 1 in Ref. [13]) of bismuth alloys. In solidification, atom attachment is slow and growth is faceted, with skeletal facet instability, overhangs and even liquid inclusion. In dissolution, atom detachment is much faster so that diffusion control is approached. Then, the habit is macroscopically smooth and rounded.

For normal growth to occur, the atoms must be able to join the solid rapidly enough so that they do not limit the process, which is not the case for AlPdMn quasicrystal growth. Indeed, the front recoil caused by the increase of the pulling velocity [arrow in Fig. 1(b)] reveals that undercooling builds up because the growth kinetics is slow due to uneasy attachment of the building elements in the melt to the quasicrystal solidification front. Indeed, as ledge limited growth is characterized by linear kinetics [26], assuming identical solutal recoil [27] the velocity jump $\Delta V$ and temperature shift $\Delta T$ between Figs. 1(a) and 1(b) can be related by the relation $\Delta V=-\mu \Delta T$ with $\mu$ the kinetic coefficient. This relation for the first time allows to directly derive a realistic estimate of the kinetic coefficient. Using $\Delta V=3.2 \mu \mathrm{m} / \mathrm{s}$ and $\Delta T=-3.5 \mathrm{~K}$ deduced from the experiment, we get $\mu=0.9 \mu \mathrm{m} \mathrm{s}^{-1} \mathrm{~K}^{-1}$. Live synchrotron observation thus shows that quasicrystal growth kinetics is actually comparable to the ledge growth kinetics of semiconductors and oxides $\left(\mu=0.826 \mu \mathrm{m} \mathrm{s}^{-1} \mathrm{~K}^{-1}\right.$ for $\mathrm{Bi}_{4} \mathrm{Ge}_{3} \mathrm{O}_{12}$ [28]), definitely much slower than the solidification kinetics of pure metals but not as sluggish as admitted. In particular, $\mu=0.9 \mu \mathrm{m} \mathrm{s}^{-1} \mathrm{~K}^{-1}$ is two orders of magnitude larger than the value derived by Dong et al. [29] using the Avrami approach of isothermal phase transformation. As this method was applied to the late stage of the transformation, this discrepancy can be attributed to the slowing down of the process with time, similarly to what is evidenced in Figs. 1(c) and 3. It is thus very much probable in Dong's experiments that the rejected aluminum, accumulated in between the numerous grains in their nearing, was continuously reducing the effective undercooling, thus increasingly poisoning the growth of the grains.

The mere fact that the nucleation of new grains does not occur during the growth sequence at $V=0.4 \mu \mathrm{m} / \mathrm{s}$ but only after the increase to $V=3.6 \mu \mathrm{m} / \mathrm{s}$ brings out fundamental information. Namely, the nucleation of new grains is enabled in the melt just above grains 1 and 2 because the kinetic undercooling needed to drive quasicrystal growth has exceeded the critical nucleation undercooling. Aside from the possibility of heterogeneous nucleation on the graphite foils that cannot be readily discarded, a very low resistance to homogeneous nucleation is anticipated due to the topological similarity between the local structural order of icosahedral AlPdMn and the icosahedral clusters in the melt $[6,30]$. This point, which is far out of the scope of the present paper, would deserve further clarification.

The asymmetry of the growth of edges 1 and 6 in Fig. 5 is the very signature of thermosolutal convection, well documented for dendrites [31]. Indeed, the rejection of both aluminum and latent heat renders the melt surrounding the solid grain lighter, thus creating a driving force for growth- 
induced natural convection. For the downward-growing edge, this buoyancy-driven convection sweeps the surrounding fluid upwards away. It follows that much undercooled melt is brought in contact with this edge, which causes it to grow faster. For the upward-growing edge, the lowundercooling fluid enveloping the grain flows upwards into the path of the edge, which causes it to propagate more slowly. Moreover, it is the impingement process of facets 4 and 5 , in which $V_{4}, V_{5}$ and concomitant aluminum and latent heat rejection together decrease to zero, that explains the second rise observed in the velocities $V_{2}$ and $V_{3}$. This rise is consequential to the decrease and cessation of the flow of the low-undercooling fluid associated to the advancing of facets 4 and 5 , upwards into the paths of facets 2 and 3.

The definitive clarification of the origin of the black contrasts decorating new grains would need a specific study, out of the scope of the present paper. Yet, tentative explanations can be proposed. As it is long established in crystal growth of semiconductors, optical crystals and oxides that the segregation of chemical species may be different on rough parts and facets (see e.g.,[32]), the most straightforward and simplest one is that these contrasts are revealing the segregation of the densest and thus most x-ray absorbing component, namely palladium, at grain edges and vertices. Otherwise, the black contrasts could be due a variation of the quasicrystal density not linked to a local variation in composition. For instance, extensive studies on garnet crystals [33] have shown that the mean lattice parameter is slightly greater in the faceted area and that this is most likely due to oxygen vacancy entrapment at the growth interface as growth steps sweep rapidly across the faceted interface. Following this line, darker edges and vertices could be denser region be- cause vacancies are similarly entrapped by the growth ledges in their rapid lateral motion over the facets. This is all the more appealing as the question of vacancies and pores is still a critical pending issue in quasicrystal solidification. It is furthermore conceivable that other explanation(s) could be postulated.

\section{CONCLUSION}

The dynamics of icosahedral AlPdMn quasicrystal growth from the melt is established by using in situ and in real-time synchrotron imaging. The faceted character of growth can be attributed to the lateral spreading of ledges. Quasicrystal growth is controlled by interface kinetics rather than by local heat flow as widely thought. A realistic estimate of the kinetic coefficient is deduced from the solid-melt interface undercooling. The growth of new grains and the process of their mutual blocking are noticeably affected by the rejection of chemical species, aluminum in the present case. Work is currently in progress at ESRF to further deepen the characterization of the solidification dynamics of quasicrystals, in particular using complementary information on growth strains and defects provided by in situ synchrotron $\mathrm{x}$-ray topography, that we have recently implemented on the experimental setup.

\section{ACKNOWLEDGMENTS}

The authors express their gratitude to T. Bactivelane and $\mathrm{R}$. Chagnon for technical assistance in the experiments. They also thank A. A. Chernov, M. Kléman, V. Dmitrienko, and M. de Boissieu for fruitful discussions.
[1] M. Boudard et al., Philos. Mag. Lett. 71, 11 (1995).

[2] Y. Yokoyama et al., Mater. Trans., JIM 38, 943 (1997).

[3] A. Langsdorf and W. Assmus, J. Cryst. Growth 192, 152 (1998).

[4] V. E. Dmitrienko, S. B. Astaf'ev, and M. Kleman, Phys. Rev. B 59, 286 (1999).

[5] C. L. Henley, Comments Condens. Matter Phys. 13, 59 (1987).

[6] V. Simonet, F. Hippert, M. Audier, and R. Bellissent, Phys. Rev. B 65, 024203 (2001).

[7] V. Elser, in Extended Icosahedral Structures, edited by M. V. Jaric and D. Gratias (Academic Press, Boston, 1989), Vol. 3, p. 105.

[8] C. Beeli and H. U. Nissen, Philos. Mag. B 68, 487 (1993).

[9] I. R. Fisher et al., Philos. Mag. B 61, 1673 (1999).

[10] J. Schroers, D. Holland-Moritz, D. M. Herlach, and K. Urban, Phys. Rev. B 61, 14500 (2000).

[11] F. Kluge et al., Surf. Sci. 519, 33 (2002).

[12] W. N. Honeyman and M. B. Small, J. Cryst. Growth 21, 155 (1974).

[13] L. M. Kolganova et al., in Growth of Crystals, edited by A. A. Chernov (Consultants Bureau, New York, 1979), Vol. 11, p. 295.

[14] P. Cloetens et al., J. Phys. D 29, 133 (1996).
[15] J. C. Labiche et al., ESRF Newsletter, Vol. 25 (European Synchrotron Radiation Facility, France, 1996) p. 41.

[16] H. Saka et al., J. Mater. Res. 20, 1629 (2005).

[17] H. Nguyen Thi et al., J. Phys. D 36, A83 (2003).

[18] O. Dovbenko, T. Velikanova, and S. Balanetskyy, in LandoltBornstein-Group IV Physical Chemistry (Springer, Berlin, 2005), Vol. 11, Subvolume A3, p. 1.

[19] H. Klein et al., J. Alloys Compd. 296, 128 (2000).

[20] See EPAPS Document No. E-PLEEE8-74-049609 for two videos showing the growth of icosahedral AlPdMn quasicrystals from the melt (synchrotron radiography at ESRF). For more information on EPAPS, see http://www.aip.org/pubservs/ epaps.html

[21] M. A. Martorano, C. Beckermann, and C. A. Gandin, Metall. Mater. Trans. A 34, 1657 (2003).

[22] A. Badillo and C. Beckermann, Acta Mater. 54, 2015 (2006).

[23] B. Willers et al., Mater. Sci. Eng., A 402, 55 (2005).

[24] R. J. Schaefer et al., Metall. Trans. A 17, 2117 (1986).

[25] A. A. Chernov, J. Cryst. Growth 24-25, 11 (1974).

[26] W. A. Tiller, The Science of Crystallization: Microscopic Interfacial Phenomena (Cambridge University Press, Cambridge, UK, 1991), Chap. 2.

[27] B. Caroli, C. Caroli, and L. Ramirez Piscina, J. Cryst. Growth 132, 377 (1993). 
[28] V. D. Golyshev, M. A. Gonik, and V. B. Tsvetovsky, J. Cryst. Growth 237-239, 735 (2002).

[29] C. Dong et al., J. Mater. Res. 6, 2637 (1991).

[30] H. Tanaka, J. Phys.: Condens. Matter 15, L491 (2003).
[31] S. H. Tirmizi and W. N. Gill, J. Cryst. Growth 85, 488 (1987). [32] J. B. Mullin and K. F. Hulme, J. Phys. Chem. Solids 17, 1 (1960).

[33] B. Cockayne, J. Cryst. Growth 42, 413 (1977). 